\title{
Observations of Motional Electromagnetic Fields During EMSLAB
}

\author{
Alan D. Chave, ${ }^{1}$ Jean H. Filloux, ${ }^{2}$ Douglas S. Luther, ${ }^{2}$ Lawrie K. Law, ${ }^{3}$ \\ AND ANTONY WHITE ${ }^{4}$
}

\begin{abstract}
The long-period ( $>1$ day) behavior of the seafloor electromagnetic fields during EMSLAB is considered in detail with an emphasis on interpretation in terms of oceanic motions. The study begins with a summary of the physics of motional electromagnetic induction, in which the seawater conductivity-weighted, vertically integrated velocity measured by the horizontal electric field is emphasized. Using frequency-domain methods, it is shown that seafloor and terrestrial magnetic variations have similar spectral shapes, indicating a common origin, but the seafloor electric field is not consistent with either at periods longer than 4 days, suggesting an oceanic source. The magnetic field variations are highly coherent across the EMSLAB array at periods shorter than 9 days, but the similarity decreases at longer periods, probably due to long-term instrumental drift. The seafloor horizontal electric field data exhibit the broadband coherence characteristic of ionospheric sources only at periods shorter than 1-2 days and are essentially incoherent between 4 days and a week except in association with certain propagating wave phenomena. The distinction between the depth-averaged velocity inferred from the horizontal electric field and its point measurement by the vertical electric field is demonstrated with data. Some specific properties of the seafloor electric fields are then considered in detail. The first of these is a 4-day wave observed to propagate from north-to-south along the east flank of the Juan de Fuca Ridge. The wavelength, propagation sense, and association with the ridge are all consistent with topographically trapped Rossby wave behavior at a zero in the group velocity. A second north-to-south propagating wave is seen at periods of 8-12 days in the middle of the EMSLAB area with a probable wavelength of order $1000 \mathrm{~km}$. Unresolved low-frequency structures in the electric field are also mentioned. These observations clearly demonstrate the power of electromagnetic array methods for the study of long-period oceanic behavior.
\end{abstract}

\section{INTRODUCTION}

Natural electromagnetic fields in the oceans are induced by both external, ionospheric and magnetospheric, electric current systems flowing at heights of $10^{2}$ to $10^{5} \mathrm{~km}$ above the Earth and by the dynamo interaction of water currents with the Earth's stationary magnetic field. The large-scale spatial and temporal morphology of the former is reasonably well understood, and the fluctuating electromagnetic fields that extemal currents produce at the seafloor can be characterized and classified. Externally induced electromagnetic fields have long been used as a source for geophysical exploration using the magnetotelluric and geomagnetic depth sounding methods.

Motionally induced electromagnetic fields are less well understood, primarily due to the paucity of observations covering the long periods (days to months) where most of the ocean's variability is concentrated. However, recent work has clearly demonstrated that electric field measurements using seafloor cables that span intense, localized streams can be interpreted in terms of fluid transport and give unique temporal information on low-frequency oceanic variability [Sanford, 1982; Larsen and Sanford, 1985]. The strong electromagnetic signature of intense eddy activity near a boundary current has been detected [Lilley et al., 1986], and an experiment to study the wavenumber structure of low-frequency

\footnotetext{
${ }^{1}$ AT\&T Bell Laboratories, Murray Hill, New Jersey.

${ }^{2}$ Scripps Institution of Oceanography, La Jolla, California.

${ }^{3}$ Geological Survey of Canada, Pacific Geoscience Center, Sidney, British Columbia, Canada.

${ }^{4}$ School of Earth Sciences, The Flinders University of South Australia, Bedford Park.
}

Copyright 1989 by the American Geophysical Union

Paper number 89JB00594

0148-0227/89/89JB-00594\$05.00 ocean variability and its relation to atmospheric forcing utilizing electromagnetic methods has been carried out [Luther et al., 1987]. Interest in applying electromagnetic principles to physical oceanographic problems is increasing rapidly.

The presence of motionally induced fields also has implications for solid earth geophysical interpretations of electromagnetic data collected at the seafloor. Oceanic contamination of the large-scale external source fields can distort magnetotelluric and geomagnetic depth sounding response functions due to the presence of both short spatial scale fields and vertical electric current modes. In addition, oceanic fields may dominate the external ones over parts of the spectrum. At the same time, an oceanographic interpretation of seafloor electromagnetic data requires some information on the electrical structure beneath the ocean due to the biasing effect of inductive and conductive interactions with the Earth. As a result, the geophysical and oceanic problems are intertwined, and an interdisciplinary approach is necessary.

In this paper, evidence for motionally induced electromagnetic fields in the EMSLAB data is considered. This begins with a review of the theory of motional electromagnetic induction that explicitly incorporates interactions with the seafloor. The spatial coherence of the offshore EMSLAB data in the frequency domain is then examined. Except at the longest resolvable periods and above $1 \mathrm{cph}$, the magnetic field is highly coherent across the EMSLAB array, and there is minimal evidence for oceanic magnetic field contamination. However, the horizontal electric field exhibits broadband coherence only at periods shorter than 1-2 days and is essentially incoherent between 4 days and the longest resolvable period except in association with certain narrow-band (in frequency space) propagating wave phenomena. The vertical electric field is nearly incoherent with the horizontal ones at the same site, showing the strong effect of depth dependence in the velocity field. Two specific characteristics of the low-frequency horizontal electric fields are also examined. The first of these is a concentration of variance at periods near 4 days for sites close to the Juan de Fuca Ridge. This feature exhibits phase propagation 
from north-to-south on the east flank of the ridge, and the available evidence suggests a topographically trapped Rossby wave as the source. The second peculiarity occurs at longer periods (8-12 days) and displays north-to-south phase propagation that is clearest midway between the Juan de Fuca Ridge and the coast of North America.

\section{MOTIONAL ELECTROMAGNETIC INDUCTION}

The framework for the theory of electromagnetic induction to be used in this paper is outlined by A.D. Chave et al. (manuscript in preparation, 1989), confirming and extending the earlier results of Sanford [1971]. This treatment is based on a separation of the electromagnetic fields into independent toroidal and poloidal magnetic (TM and PM) modes under the assumption that the electrical conductivity of the ocean and Earth varies only vertically. Using a set of Green functions valid for an ocean of constant conductivity and depth overlying a layered earth, an exact set of integral equations are derived which describe the electromagnetic fields in an ocean of vertically varying conductivity. Some characteristics of the real ocean allow important simplifications to be made at periods substantially longer than an inertial day, including the following (1) the horizontal length scale is typically large compared to the depth of the ocean and (2) the vertical velocity is negligible compared to the horizontal components. It is also assumed that the effect of self-induction is not large. In addition, the geomagnetic field is modeled as an inclined geocentric dipole. Using these conditions, approximate solutions to the integral equations are constructed; these are summarized here.

Consider a Cartesian coordinate system with $\hat{z}$ positive upward and zero at the ocean's surface. The motional horizontal electric field is independent of depth and may be written

$$
\mathbf{E}_{h}(\boldsymbol{\rho})=C F_{z} \hat{z} \times\left\langle\mathbf{v}_{h}\right\rangle^{*}+\mathbf{N}
$$

where

$$
\left\langle\mathbf{v}_{h}\right\rangle^{*}=\frac{1}{\langle\sigma\rangle H} \int_{-H}^{0} d z^{\prime} \sigma\left(z^{\prime}\right) \mathbf{v}_{h}\left(\rho, z^{\prime}\right)
$$

is the conductivity-weighted, vertically integrated horizontal water velocity, $H$ is the water depth, $\langle\sigma\rangle$ is the depth-averaged conductivity, $C$ is a scale factor which depends on the electrical conductivity structure beneath the seafloor, $\rho=x \hat{x}+y \hat{y}, F_{z}$ is the vertical component of the geomagnetic induction, and $\mathbf{N}$ is a small error term that depends on the velocity field over a wide area as well as random effects. The multiplicative relation with $C$ in (1) is more properly a convolution relation between a spatial averaging kernel and (2), with an averaging distance in the horizontal that is of order a couple of times the water depth. This means that the horizontal electric field is actually a spatial average of the water velocity. $C$ can be approximated as a constant slightly smaller than 1 for realistic conductivity models of the Earth and may be computed from contemporaneous electric field and conventional measurements of the water velocity. $\mathbf{N}$ represents the influence of nonlocal motional electric currents and is discussed by Sanford [1971] and A.D. Chave et al. (manuscript in preparation, 1989). Its magnitude is generally small compared to the first term in (1). The remaining part of $\mathbf{N}$ is due to geomagnetic noise and is quantified in this paper. Due to the seawater conductivity weighting in (2), the horizontal electric field (1) is a filtered version of the water velocity which accentuates the depthindependent (barotropic) component and contains limited contamination from the depth-dependent (baroclinic) part. In fact, (2) reduces to the barotropic velocity when $\mathbf{v}_{h}$ is independent of depth. A seafloor cable measures the horizontal integral of (1); see Sanford [1982] for a discussion of cable measurement interpretation.

The expression for the vertical electric field is much simpler than (1). Rewriting the vertical component of Ohm's law for a moving medium in the form

$$
E_{z}(\boldsymbol{\rho}, z)=-\left[\mathbf{v}_{h}(\boldsymbol{\rho}, z) \times \mathbf{F}_{h}\right] \cdot \hat{z}+J_{z}(\boldsymbol{\rho}, z) / \sigma
$$

it can easily be shown that the vertical electric current density term is negligible compared to the motional term if the electrical conductivity of the seafloor is substantially smaller than that of seawater. This means that the vertical electric field is proportional to the horizontal water velocity at the point of measurement, as given by the first part of (3). Since the geomagnetic field is almost axial, $E_{z}$ gives the local geomagnetic east-west water velocity; this is nearly the zonal component at mid- to lowlatitudes.

By procedures similar to those used to get (1), an expression for the motional horizontal magnetic field can be obtained. The horizontal magnetic field varies with depth because electric currents flow in different directions at different points in the ocean and in the earth, and the magnetic field is a weighted spatial average of them. An expression similar to (1) involving both local and nonlocal electric currents that are proportional to the conductivity-weighted, vertically integrated water velocity (2) is derived in A.D. Chave et al. (manuscript in preparation, 1989). However, the relative balance of the two contributions is not simple, and the interpretation of the motional horizontal magnetic field depends in a complex way on the electrical structure of the earth and the vertical distribution of seawater conductivity and water velocity. The motional horizontal magnetic field is proportional to several small physical parameters, unlike the electric field, and is expected to be especially weak. The vertical magnetic field is due entirely to nonlocal electric currents and behaves qualitatively like the horizontal components except that it is depth-independent. Due to this complexity, the motional magnetic fields will not be quantified here.

In summary, motional horizontal electric fields at subinertial periods where the horizontal length scale of an ocean flow is typically much larger than the water depth are proportional to the cross product of the vertical component of geomagnetic induction with the conductivity-weighted, vertically integrated horizontal water velocity. The constant of proportionality depends on the electrical conductivity profile beneath the seafloor and approaches unity as the seafloor becomes more resistive. This means that the north electric field component is a measure of the velocity to the west, while the east electric field is a measure of the velocity to the north in the northern hemisphere. The conductivity weighting of the velocity in (2) may be quantified using historic temperature and salinity information to construct conductivity profiles but generally is dominated by the barotropic velocity. This is a quantity that is exceptionally difficult to measure by conventional means (e.g., mechanical current meters) since the baroclinic velocity field is typically more energetic than the barotropic part in the world oceans. By contrast, the vertical electric field expression (3) is much simpler, and $E_{z}$ gives the geomagnetic east-west water velocity due to barotropic and baroclinic flows at the measurement point. Motional magnetic fields are substantially more complicated, involving weighted averages of both local and nonlocal electric currents. 
DATA

A description of the EMSLAB experiment, including the goals and experimental layout, is given by EMSLAB Group [1988]. In this paper, only the offshore phase of the project will be considered. A total of 39 bottom packages were deployed at 19 sites, and all except one unit were recovered; usable data were returned by 33 of the instruments. The time series selected for this study consist of the electric and magnetic ones collected by Scripps Institution of Oceanography (SIO), the magnetic results of the Pacific Geoscience Center (PGC), and a single magnetic field record obtained by Flinders University of South Australia (FUA). The corresponding locations for the instruments are shown in Figure 1 along with selected bathymetric contours. All of the instruments are from SIO except for sites SE2, SE6, SF1, and SG5 (PGC), and site SF2 (FUA); see Figure 1 for site information. In addition, standard observatory data from Victoria, British Columbia, Canada $\left(48^{\circ} 31^{\prime} \mathrm{N}, 123^{\circ} 25^{\prime} \mathrm{W}\right)$ were obtained for reference purposes.

The seafloor instruments consist of four fundamental types. The horizontal electric field sensors, or horizontal electrometers (10 packages), measure the electric potential between two orthogonal pairs of points in the horizontal plane and separated by about $6 \mathrm{~m}$. Contact between the ocean and sensor $\mathrm{Ag}-\mathrm{AgCl}$ electrodes is made via a saltwater bridge and through a water chopper to separate electrode noise from the electric field signal. The chopping technique preserves a true zero datum, so that the measured electric field is physically meaningful down to $\mathrm{dc}$. The resolution of the electrometers is $0.015 \mu \mathrm{V} \mathrm{m}^{-1}$, and the sampling rate is 128 $\mathbf{h}^{-1}$. Further details are given by Filloux [1987]. Two vertical

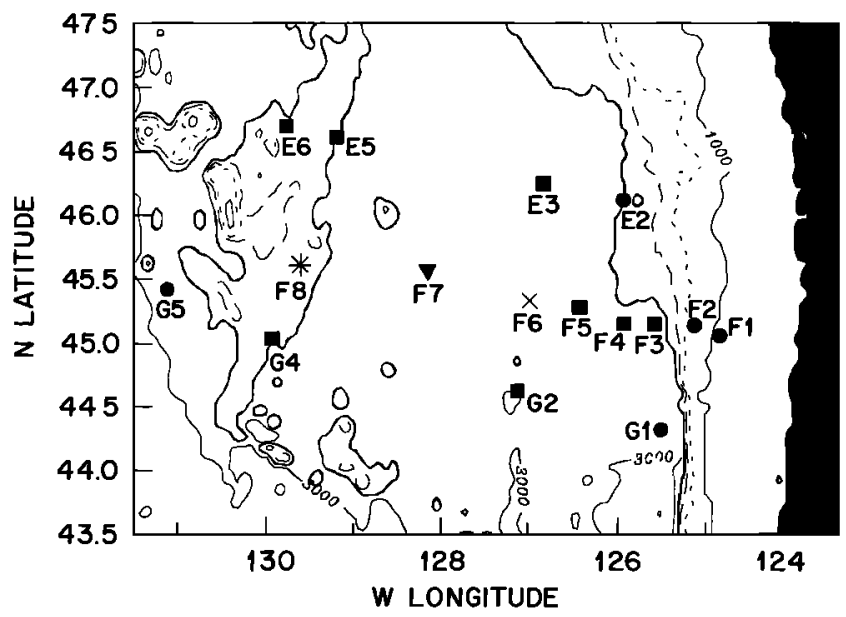

Fig. 1. The locations of the seafloor instruments used in this study superimposed on selected bathymetric contours taken from the ETOPO5 digital data base compiled by the National Geophysical Data Center. The seafloor sites from EMSLAB are denoted by a prefix $S$ (suppressed on the map) and arranged in three roughly east-west lines designated by a second letter $\mathrm{E}, \mathrm{F}$, or $\mathrm{G}$ starting at the north. The stations are then numbered beginning at the coast. Starting at the northeast comer, the sites shown are SE2, SE3, SE5, and SE6 (north line), SF1, SF2, SF3, SF4, SF5, SF6, SF7, and SF8 (middle line), and SG1, SG2, SG4, and SG5 (south line). The symbols indicate combined horizontal electric and three-component magnetic sites (solid squares), three-component magnetic sites (solid circles), a vertical electric field site (cross), a three-component electric and magnetic site (solid triangle), and a horizontal electric field site (star). The contours shown are the 3000-m (labeled), 2600-m (heavy line), 2200-m (long dashed line), 1800-m (short dashed line), and 1000-m (labeled) ones; the North American coast is the solid area to the right. The broad, flat region in the middle is the Cascadia Basin, while the $2600-\mathrm{m}$ contour at the left outlines the Juan de Fuca Ridge.
TABLE 1. Tidal Lines and Periods

\begin{tabular}{cc}
\hline Species & Period \\
\hline$M_{f}$ & 327.858987 \\
$2 Q_{1}$ & 28.006219 \\
$Q_{1}$ & 26.868354 \\
$O_{1}$ & 25.819339 \\
$M_{1}$ & 24.849159 \\
$S_{q}^{0}$ & 24.000000 \\
$J_{1}$ & 23.098475 \\
$O O_{1}$ & 22.306072 \\
$2 N_{2}$ & 12.905373 \\
$N_{2}$ & 12.658347 \\
$M_{2}$ & 12.420605 \\
$L_{2}$ & 12.191619 \\
$S_{q}^{1}$ & 12.000000 \\
$S_{q}^{2}$ & 8.0000000 \\
$S_{q}^{3}$ & 6.0000000 \\
$S_{q}^{4}$ & 4.8000000 \\
$S_{q}^{5}$ & 4.0000000 \\
\hline
\end{tabular}

electrometers placed about $100 \mathrm{~m}$ above the seafloor were also used. One of these was an older unit measuring the potential between the ends of a 100 -m-long vertical wire and is described by Bindoff et al. [1986]. The second was a prototype unit using an adaptation of the horizontal electrometer water chopper and covered by Filloux [1987].

The SIO magnetometers are modern versions of suspended magnet systems using extremely rigid and stable suspension fibers and optoelectronic feedback nulling. These units have a resolution of $0.2 \mathrm{nT}$ and sample the magnetic field at a rate of $64 \mathrm{~h}^{-1}$. The PGC magnetometers are standard fluxgate designs with a resolution of $1 \mathrm{nT}$ and a sample rate of $30 \mathrm{~h}^{-1}$. The FUA magnetometer is similar to the PGC units and is described by White [1979]. Further information on seafloor magnetometers is given by Filloux [1987].

All of the time series were carefully edited to remove bad values using standard methods. Because of the water chopper, the horizontal electric and chopper-type vertical electric data required no trend removal, and no further preprocessing was necessary. The data from the long-wire vertical electrometer (site SF6) displayed serious drift at periods longer than about a day and were not used in the subsequent analysis. Most of the SIO magnetic data showed long-term exponential drift caused by settling of the instrument package into the sediments on the seafloor. This was corrected when necessary by least squares removal of an exponential trend. The PGC and FUA magnetometers were less sensitive to package motion, but some of the time series displayed drift problems that were traced to magnetization of lithium batteries on discharge. This was detrended by fitting a least squares, variable knot spline function with two to three free knots.

All of the raw time series are dominated by high-amplitude, periodic signals due to the solar daily variation, its harmonics, and the ocean tides. Prior to further analysis, the components listed in Table 1 were removed by robust least squares [Chave et al., 1987]. The lines listed in the table consist of $S_{q}$ with its first six harmonics and the largest tidal components that are separated by the Rayleigh resolution criterion (i.e., their frequencies differ by at least the inverse time series length). While superresolution of 
more closely spaced lines was sometimes feasible, especially with the lower noise SIO data, theory shows that this depends critically on the unknown signal-to-noise ratio and hence could not be quantitatively assessed. For archival purposes, Tables A1 through A6 on microfiche ${ }^{1}$ list the time series mean, initial and final data variances, and the resulting amplitude, percent error at the 95\% level, phase, and its $95 \%$ uncertainty for each component. The phases are referenced to 0000 UT on January 1, 1985, and the convention used for the periodic components is $A_{t} \cos \left(\omega_{t} t+\phi_{t}\right)$, where $A_{t}$ is the line amplitude and $\phi_{t}$ is the phase at angular frequency $\omega_{l}$. Independent and identically distributed regression residuals are a condition of the Gauss-Markov theorem from which error estimates are derived; they must also be Gaussian to get confidence limits. Use of a robust least squares procedure is essential to obtaining meaningful confidence limits on the amplitudes and phases because of the dramatically non-Gaussian form the residuals from natural source electromagnetic data take without one.

Previous studies have shown that the ocean tides produce detectable electromagnetic effects in seafloor data [e.g., Larsen, 1968]. For these data, the horizontal electric field is dominated by $S_{q}$ and its first three harmonics and the $M_{2}$ lunar semidiurnal tide, although the $Q_{1}, O_{1}$, and $N_{2}$ constituents are also significant. The vertical electric field measurement displays sizable diurnal and semidiurnal ocean tides with no $S_{q}$ harmonic components. The periodic terms in the magnetic field are largely due to $S_{q}$ with its first two harmonics and $M_{2}$. It should be noted that the large $K_{1}$ diurnal ocean tide differs in frequency by only one cycle per year from $S_{q}^{0}$, so that estimates for the latter are probably contaminated by an unresolvable component. A similar argument applies to $S_{q}{ }^{1}$ and the $P_{2}$ and $K_{2}$ tides, although the latter are much smaller than $K$.

A detailed examination of the tidal components is beyond the scope of this paper. The line parameter estimates are provided here for use in other studies. After removal of the tides, all horizontal time series were rotated to a geographic coordinate system. The data were then low-passed with a prolate filter having a 3-dB point at $2 \mathrm{cph}$ and $60 \mathrm{~dB}$ attenuation in the stopband and decimated to four samples per hour for further analysis.

\section{SPECTRAL ANALYSIS}

A major problem in time series analysis is the choice of an estimation procedure that yields a spectral estimate from a finite observation of the process of interest that is not badly biased yet remains statistically consistent and efficient. This problem becomes especially acute when the time series is short (in the sense that the interesting phenomena have characteristic frequencies of order the inverse sequence length), contains periodic components, or has a large dynamic range. For the offshore EMSLAB data, the periodic signals have known frequencies and are easily removed using the methods of the last section. However, these data series are typically 60 days in length, and the period range of interest in oceanography goes from a few days to many months.

The standard approach to such problems in geophysics has traditionally been a band-averaging one, in which the entire time series is multiplied by a data taper, Fourier transformed, and

\footnotetext{
${ }^{1}$ Appendix tables are avallable with entire article on microfiche. Order from American Geophysical Union, 2000 Florida Avenue, N.W., Washington D.C. 20009. Document B89-029; \$2.50. Payment must accompany order.
}

weighted averages over adjacent frequencies are computed to get a consistent spectrum. There are serious problems with this method, principally because of an inability to utilize a low bias data window without dramatically decreasing the variance efficiency. Band-averaged estimates may be badly biased, usually in a manner that is misleading and difficult to detect, and should be regarded as obsolete.

A modern replacement for band-averaged spectra based on a multiple prolate window expansion of the Fourier transform was introduced by Thomson [1982] and will be used throughout this paper. The multiple window approach generalizes the role of a data taper and involves the separate processing of a time series using each member of a set of orthogonal data windows that are optimal in a minimum spectral leakage sense and which depend on a free parameter, the resolution bandwidth or time-bandwidth product. Each of these raw estimates is essentially independent, and the final result is a weighted average of the individual spectra and hence is statistically consistent. This gives high variance efficiency with good control of the bias caused by spectral leakage. The final spectrum is effectively the convolution of the unknown true spectrum with a rectangular frequency-domain window of width governed by the time-bandwidth product. Further information on the multiple window method is given by Thomson [1982]. Certain normalizations used here differ slightly from Thomson's formulation; see F.L. Vernon et al. (manuscript in preparation, 1989) for details.

The horizontal electric field (1) is a measure of the vector horizontal velocity, and a Cartesian approach to its analysis has several drawbacks. First, a complete treatment of two sets of horizontal velocity measurements involves four sets of coherences and phases for each frequency, each of which has similar significance. Second, the coherences are not invariant under coordinate rotation, and local effects that change the natural coordinates for different data series can produce misleading results. An alternate approach based on a rotary representation is in common use by oceanographers and is discussed by Mooers [1973]. A horizontal velocity vector at a single site is represented as a complex quantity and decomposed into two counterrotating circular components with distinct amplitudes and phases. This means that spectra may be different for positive and negative frequencies. The convention chosen here is that anticlockwise motions correspond to positive frequencies and clockwise motions correspond to negative ones. Because of the circular symmetry, coordinate invariance holds for the coherence, while the phase changes in proportion to any relative coordinate rotation; the differential coordinate system orientation can also be obtained from combinations of the rotary crossspectra. This makes it possible to quickly determine the statistical relationships between sets of vector time series. In addition, there are strong dynamical reasons to prefer the rotary form for spectra of ocean velocity because some types of wave disturbances will be preferentially polarized in a clockwise sense due to rotation of the Earth.

\section{SPECTRA OF THE ELECTROMAGNETIC FiIELDS}

Multiple window power spectra for all of the seafloor electric and magnetic components were computed as a summary representation of the data. After prewhitening with a five-term autoregressive filter, spectra with a time-bandwidth product of four and eight windows were obtained and corrected for the prewhitening, yielding approximately 16 degrees-of-freedom per frequency. This gives a double-sided $95 \%$ confidence limit on the spectrum of $(0.6,2.3)$ times the estimate at each frequency, assuming $\chi^{2}$ statis- 

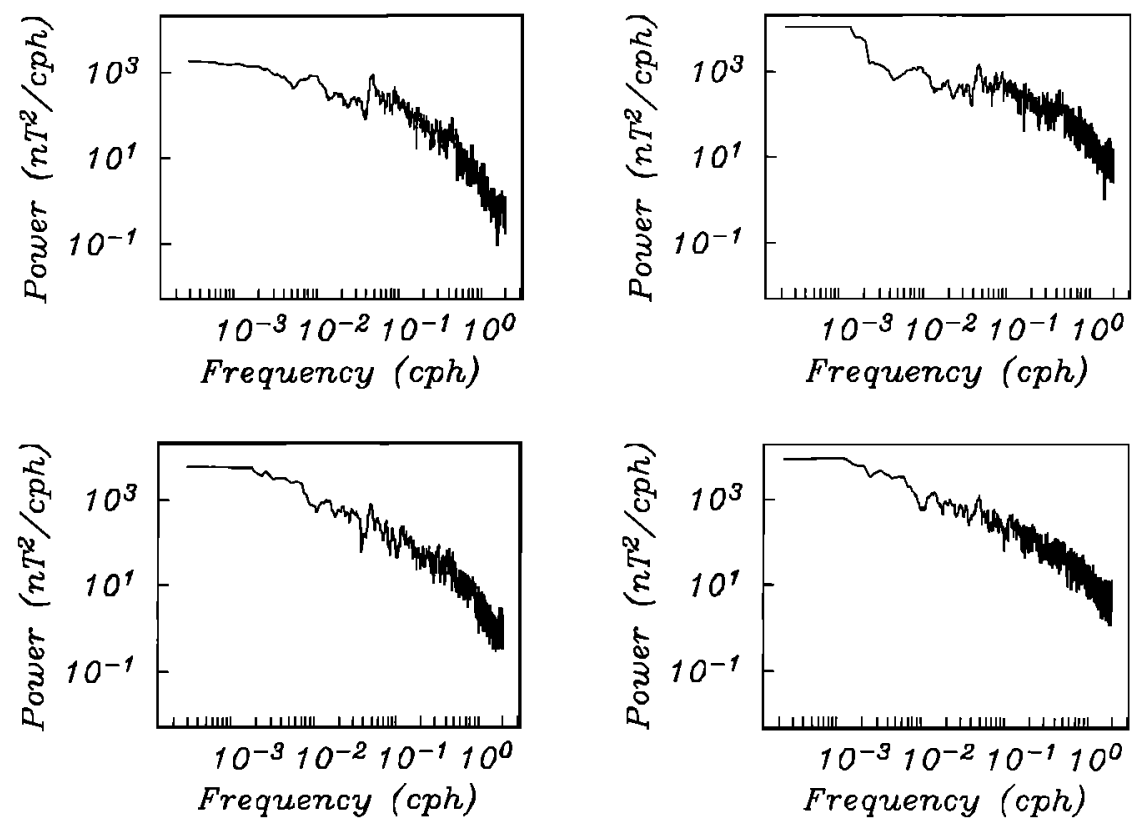

Fig. 2. Multiple prolate window power spectra for the horizontal magnetic field variations at (left) seafloor site SE3 and (right) the standard observatory at Victoria, British Columbia, Canada, for the EMSLAB epoch. The geographic north components are shown in the bottom two panels, while the geographic east ones are at the top. The spectra have a time-bandwidth product of 4 and were computed with eight orthogonal data windows, yielding about 16 equivalent degrees-of-freedom per frequency for $95 \%$ confidence limits of $(0.6,2.3)$ times the estimate. The spectral bandwidth is $\approx 0.0028 \mathrm{cph}$.

tics. Since there are about 60 days or 1440 hours of data available, the spectral bandwidth is $=0.0028 \mathrm{cph}$; features that are more closely spaced than this are not clearly resolvable.

Figure 2 compares power spectra of the horizontal magnetic field components at site SE3, located near the middle of the Cascadia Basin, to corresponding spectra for Victoria Observatory. The remaining seafloor magnetic field spectra are qualitatively similar to those at SE3, and no distinct features could be detected at individual sites. The primary characteristic distinguishing the seafloor and land spectra is the marked attenuation of the seafloor magnetic field at frequencies above $0.1 \mathrm{cph}$ caused by the conductive ocean layer. Expressions quantifying this reduction in power are given by Chave and Filloux [1984], and it can amount to a decade or more at $1 \mathrm{cph}$. However, at periods longer than a day, the spectra do not differ significantly except for the increase in power at the lowest resolvable frequencies for the east component at Victoria. This is caused by a slight trend of probable instrumental origin in the data and is not important. Note that the spectra are redder at high frequencies than at low ones with a break in slope at about $1 \mathrm{cpd}$. The seafloor east magnetic field spectra are nearly white at frequencies below $1 \mathrm{cpd}$, while the slopes of the north ones are roughly proportional to inverse frequency in that region. Spectra of the vertical magnetic field (not shown) are qualitatively similar to those for the east component except for a reduction in power by up to a factor of 10 , reflecting the weaker vertical magnetic variation field.

Figure 3 shows power spectra of the electric fields at sites SE3, located near the center of the Cascadia Basin, and SG4, located on the southeast flank of the Juan de Fuca spreading center (see Figure 1). Land electric field data that are usable to the low frequencies considered here were not collected during EMSLAB, so no terrestrial comparison is possible. Under the quasistatic approximation to the Maxwell equations, in which displacement currents are neglected, the electric field is proportional to the time deriva- tive of the magnetic field. Since the electric field is not appreciably attenuated by the ocean layer at frequencies below $1 \mathrm{cph}$, the seafloor electric spectra should be compared to the land magnetic ones. This means that electric field spectra should be similar to their terrestrial magnetic field counterparts scaled by the frequency squared. Comparing Figures 2 and 3 shows that this is approximately true at frequencies above $0.01 \mathrm{cph}$ (4-day period). The electric field spectra are red (decreasing with rising frequency) above $0.3 \mathrm{cph}$, white between 0.3 and $0.04 \mathrm{cph}$ (1-day period), and blue (decreasing with falling frequency) from 0.04 to $0.01 \mathrm{cph}$, consistent with Figure 2 (right panels) scaled by frequency squared. However, the electric field spectra rise dramatically at frequencies below $0.01 \mathrm{cph}$ in a manner that is not predictable from Figure 2, suggesting a source for the electric field that does not significantly influence the magnetic field. It is a central assertion of this paper that the dramatic low-frequency rise in power is due to motional induction, and the remainder of the work will document the consistency of this hypothesis. Note that the hole in the north electric spectrum at frequencies between 0.005 and $0.05 \mathrm{cph}$ is deeper than that in the east ones, as expected from the smaller spectral slope of the corresponding east and north magnetic spectra in Figure 2. Note also the large spectral bump in the site SG4 result near $0.01 \mathrm{cph}$; this is especially strong in the east component but is also apparent in the north one. It will be shown later that this is due to a propagating wave trapped to the Juan de Fuca Ridge.

Figure 4 shows a power spectrum for the vertical electric field at site SF7. This component is entirely of oceanic origin in the absence of marked lateral heterogeneity of electrical conductivity. The solid line shows a model spectrum for the vertical electric field produced by ambient internal waves that is based on the Garrett-Munk kinematic description, as derived by Chave [1984]. Agreement with the data at frequencies between 0.06 and $1 \mathrm{cph}$ is within the uncertainties except near the inertial frequency $(\approx 0.058$ 

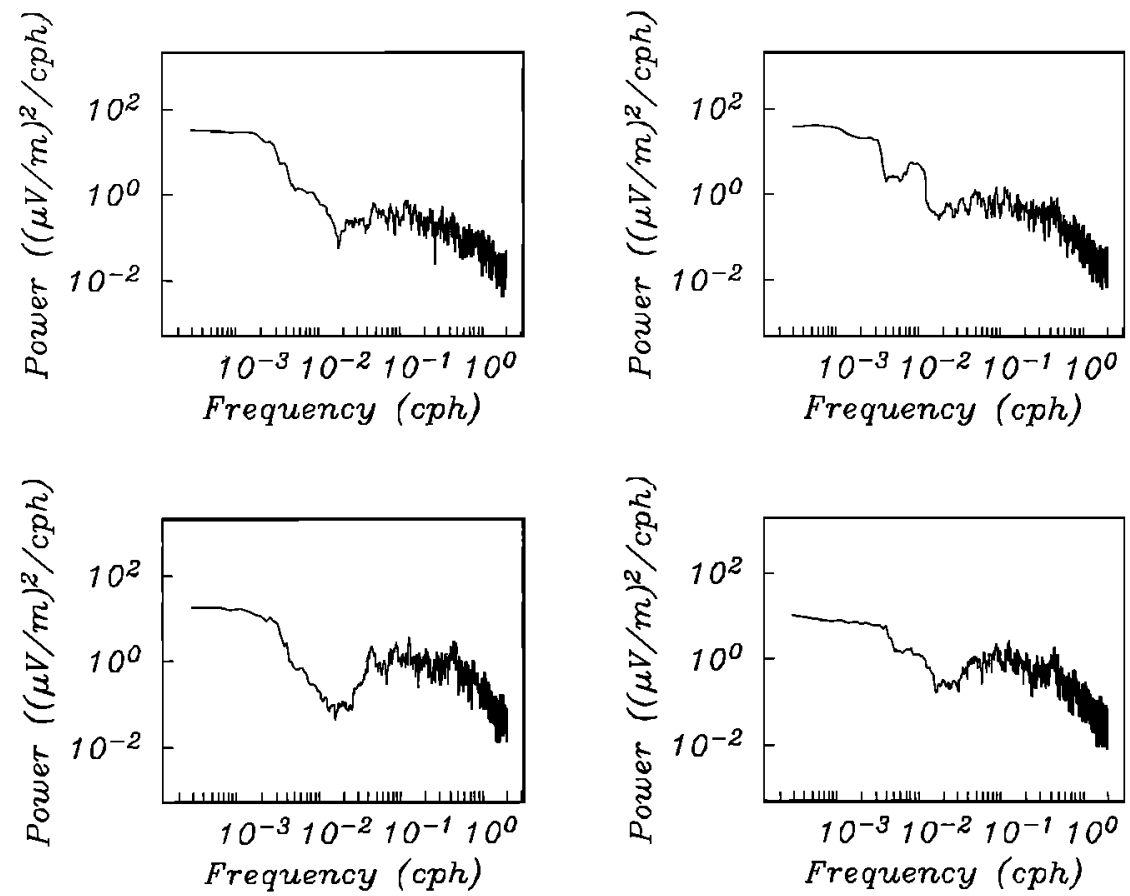

Fig. 3. Multiple prolate window power spectra for the horizontal electric field variations at seafloor sites (left) SE3 and (right) SG4 for the EMSLAB epoch. The geographic north components are shown at the bottom, while the geographic east ones are at the top. The spectra have a time-bandwidth product of 4 and were computed with eight orthogonal windows, yielding about 16 equivalent degrees-of-freedom per frequency for $95 \%$ confidence limits of $(0.6,2.3)$ times the estimate. The spectral bandwidth is $\approx 0.0028$ cph.

cph), where the model predicts an excess of variance. Previous comparisons of seafloor vertical electric field observations and the intemal wave spectrum show similar consistency [Chave and Filloux, 1985; Bindoff et al., 1986]. The large peak at 0.06-0.1 cph in Figure 4 is due to inertial waves whose characteristic vertical eigenfunctions are strongly depth dependent [e.g., $F u, 1981]$. These are not seen in Figure 3 because the inherent vertical averaging of the horizontal electric field smooths out their effect. From (3), the vertical electric field spectrum can be converted to units of $\mathrm{cm}^{2} \mathrm{~s}^{-2} \mathrm{cph}^{-1}$ by scaling with a factor of 22.7. The remaining, subinertial range in Figure 4 is qualitatively consistent

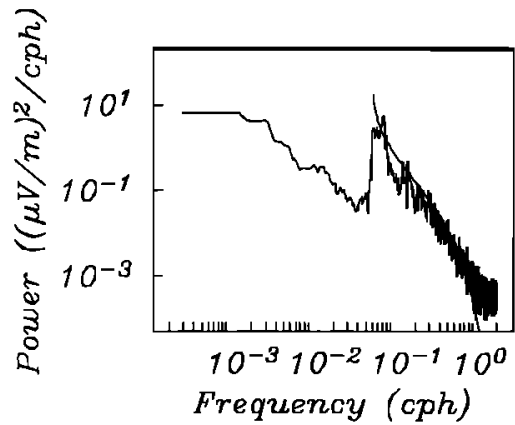

Fig. 4. Multiple window power spectrum for the vertical electric field variations at site SF7 (solid triangle in Figure 1). The spectrum has a time-bandwidth product of 4 and was computed with eight orthogonal windows, yielding about 16 equivalent degrees-of-freedom per frequency for $95 \%$ confidence limits of $(0.6,2.3)$ times the estimate. The spectral bandwidth is $\approx 0.0028 \mathrm{cph}$. The solid line is a model spectrum for the vertical electric field induced by oceanic internal waves as derived by Chave [1984]. with mechanical current meter spectra for the zonal water velocity obtained at similar latitudes; see Wunsch [1981] for some examples.

In summary, the seafloor and land magnetic spectra are similar except for the well-understood high-frequency attenuation of the former by the conducting ocean. By contrast, seafloor horizontal electric spectra contain two different components, one of which is consistent with the magnetic spectra and one that is clearly due to a new process. To more clearly delineate the source of the new contribution, it is necessary to use multivariate spectral techniques.

\section{COHERENCE Structure of THE MAgnetic Field}

Estimates for the two-point and multiple squared coherences (hereafter simply called the coherences) between the seafloor electric or magnetic fields and both the magnetic field at Victoria Observatory and other seafloor measurements were computed using the multiple prolate window method following F.L. Vernon et al. (manuscript in preparation, 1989). After prewhitening each data sequence with a five-term autoregressive filter, 13 windowed raw estimates with a time-bandwidth product of 6.5 were obtained, combined using adaptive weighting, and corrected for the prewhitening to get the coherence. These quantities possess about 26 degrees-of-freedom at each frequency, so the zero coherence level is 0.22 for the ordinary coherence and 0.26 for the multiple coherence with three input terms assuming normally distributed data; values smaller than these are indistinguishable from zero. The spectral bandwidth is $\approx 0.0045 \mathrm{cph}$.

Figure 5 shows the multiple coherence between the individual seafloor magnetic field components at site SF7, located near the middle of the Cascadia Basin, and all three magnetic field com- 

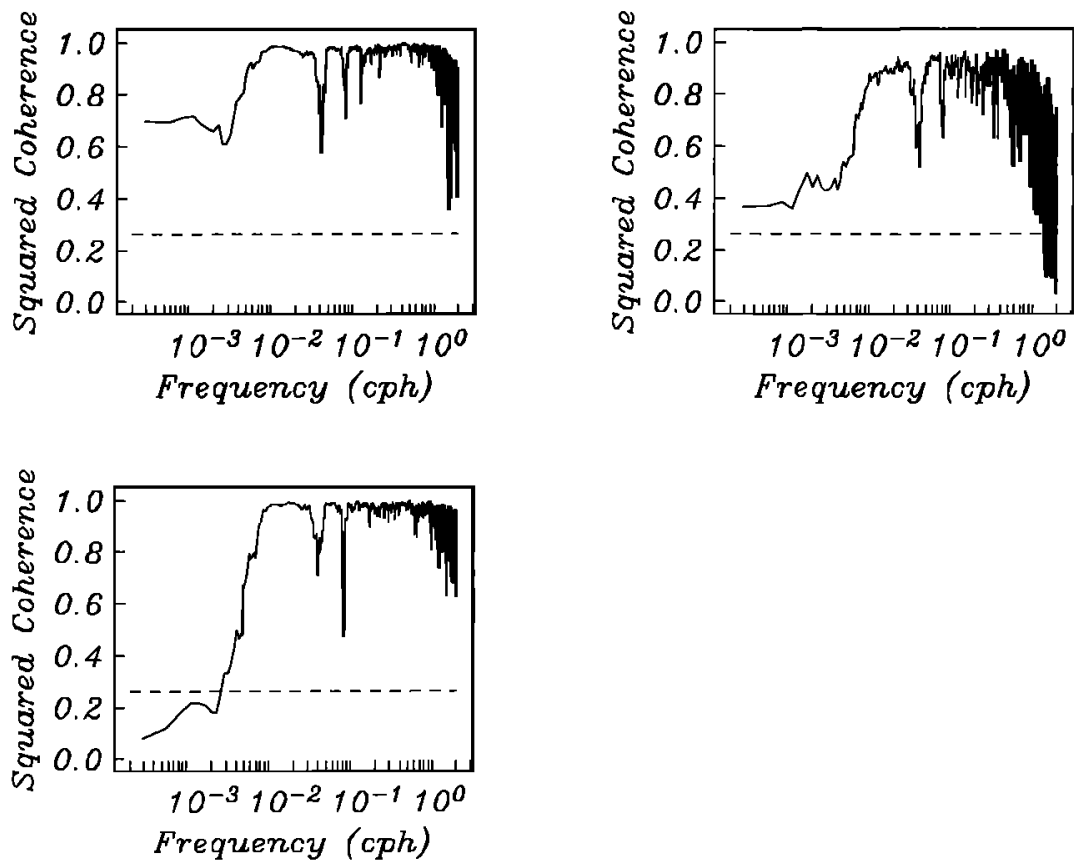

Fig. 5. Multiple window multiple coherences between the individual magnetic field components at seafloor site SF7 with all three magnetic field components at Victoria Observatory. Clockwise from the lower left, the geographic north, east, and vertical components are featured. The coherences have a time-bandwidth product of 6.5 and were computed with 13 orthogonal windows, yielding about 26 equivalent degrees-of-freedom per frequency. The zero coherence level is shown by the horizontal dashed line, while the bandwidth of the estimate is $\approx 0.0045 \mathrm{cph}$.

ponents at Victoria Observatory. The coherence typically exceeds 0.95 and is often over 0.99 from $\approx 0.005$ to $1 \mathrm{cph}$. The falloff at high frequencies is due to attenuation of the seafloor magnetic fields and a consequent increase in the relative noise level. Holes in the coherence spectrum are obvious at the frequency of $S_{q}$ and its first two harmonics, presumably due to a residual component that contains a substantial short spatial scale part that is not eliminated by the least squares line removal procedure. The lowfrequency falloff in coherence occurs only for frequencies within the resolution bandwidth, and its magnitude is both componentand site-dependent. This means that it could be due to either unresolved low-frequency contamination of the seafloor magnetic field by the ocean or to errors induced by instrumental drift and the process of detrending. Note that the low-frequency coherence is not significant for the north magnetic field component but is significant but reduced for the remaining ones. The multiple coherences for the magnetic fields at other seafloor sites and Victoria Observatory are qualitatively similar.

The two-point coherences for a single magnetic field component at two seafloor sites were computed for all station pairs. The general features seen in Figure 5 are confirmed; in addition, the magnetic fields are in phase at frequencies below $0.3 \mathrm{cph}$ throughout the array when the coherence is significant. The coherence is somewhat higher as the station separation decreases and can exceed 0.99 for closely spaced measurements. The drop in coherence within a spectral bandwidth of zero frequency is also confirmed, with no consistent pattern to the change being detectable. The holes in the coherence at the frequencies of $S_{q}$ and its harmonics depend strongly on the orientation of the two stations, being large when the latitude separation is substantial and vanishing for two sites located at the same geomagnetic latitude. There are also substantial changes in the relative phases at frequencies above $0.3 \mathrm{cph}$ that are certainly due to electrical structure changes and source field variations. A detailed discussion of such effects will not be given in this paper.

In conclusion, the seafloor magnetic field is highly coherent across the EMSLAB array at periods shorter than 9 days. This is the longest period which can be reliably studied (in a statistical sense) with this short data set. A decrease in coherence is observed at longer periods, often to a zero value, but no clear spatial pattern can be detected. The evidence for motional magnetic fields in these data is equivocal, with the low-frequency falloff in coherence being suggestive but not clearly differentiable from contamination by long-term trends in the data. There is no indication of motional magnetic fields at periods shorter than 9 days. The high coherence of the magnetic field components, including the vertical one, to fairly long periods suggests that the geomagnetic depth sounding method could profitably be employed on the seafloor. If very low frequencies can be achieved, then studies like that of Schultz and Larsen [1987] could gain more global coverage.

\section{COHERENCE STRUCTURE OF THE ElECTRIC FIELD}

Figure 6 shows the multiple coherence between the north and east electric field components at SF7 and all three magnetic field components at Victoria Observatory and at the same site. The coherences are qualitatively similar, with a value of $\approx 0.9$ from a frequency of $\approx 0.04 \mathrm{cph}$ to the Nyquist value and a sharp falloff below this. The coherence with the local magnetic field falls off faster near the upper frequency limit due to the weakness of the seafloor magnetic field and a concomitant rise in the relative noise level. As for Figure 5, holes in the coherence at the frequencies of $S_{q}$ and its first few harmonics are apparent. Neither set of coherences is significant at frequencies below $0.01 \mathrm{cph}$ (4-day period) except for a slight bulge in the east electric component centered at 

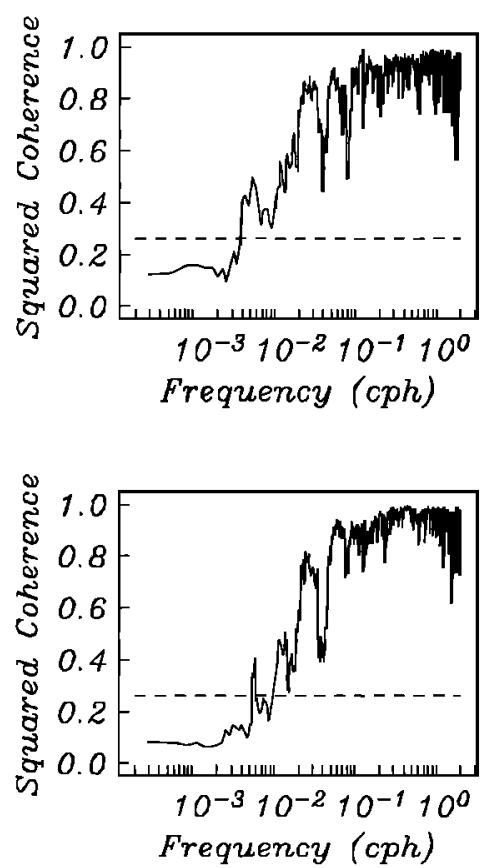
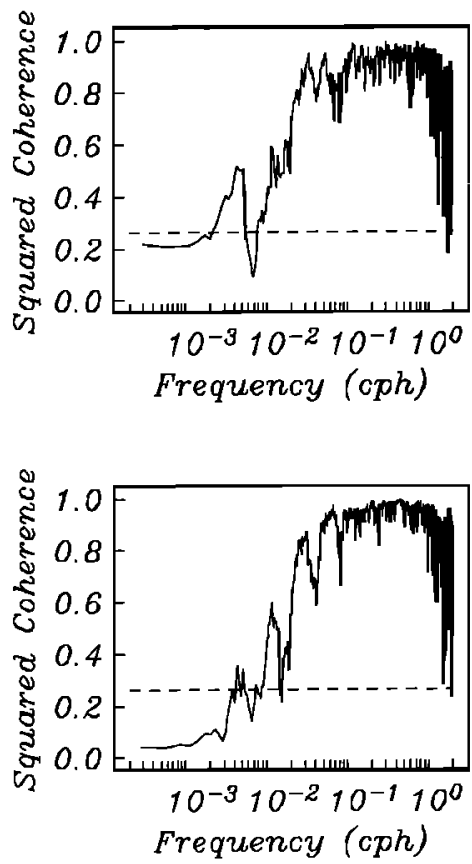

Fig. 6. Multiple window multiple coherences between the horizontal electric field components at seafloor site SF7 and all three magnetic field components at (left) Victoria Observatory and (right) site SF7. The geographic north electric component is considered in the bottom panels, while the geographic east one is shown at the top. The coherences have a time-bandwidth product of 6.5 and were computed with 13 orthogonal windows, yielding about 26 equivalent degrees-of-freedom per frequency. The zero coherence level is shown by the horizontal dashed line, while the bandwidth of the estimate is $\approx 0.0045 \mathrm{cph}$.

$0.004 \mathrm{cph}$. The existence of this peak is sensitive to the inclusion of magnetic storms in the data set being analyzed; if their effect is eliminated by using a robust estimator [Chave et al., 1987], it vanishes, suggesting an origin in the storm-time part of the ring current. This is expected to appear predominantly in the east electric and north magnetic fields. The falloff in coherence between the seafloor electric and magnetic fields at periods longer than a few days has been previously documented, and combined with the rapid high-frequency roll-off above $1 \mathrm{cph}$, it results in a bandlimited spectrum for magnetotelluric analysis.

If an oceanic electric field measurement is to serve as a substitute for a direct velocity measurement, it is necessary to find the relevant scale factor to get the correct units. For the vertical electric field, the conversion is trivial; dividing it by the north geomagnetic field and changing sign gives the geomagnetic east velocity. For the EMSLAB area, the north component of $\mathbf{F}$ is about $21,000 \mathrm{nT}$, so the scale factor is 4.76 to get velocity in $\mathrm{cm}$ $\mathrm{s}^{-1}$ from the electric field in $\mu \mathrm{V} \mathrm{m}^{-1}$.

For the horizontal electric field and in the absence of a correction for conductive interactions with the Earth, division by the vertical geomagnetic field gives an upper limit to the water velocity. For the EMSLAB area, the vertical geomagnetic field is about $50,000 \mathrm{nT}$, and a scale factor of 2.00 yields velocity in $\mathrm{cm} \mathrm{s}^{-1}$. This will be reduced by the effect of current leakage into the Earth. Proper correction of the horizontal electric field requires either a detailed knowledge of the Earth's conductivity structure or an independent measure of the water velocity. The former is difficult to achieve because shallow, low-conductivity zones are especially important in modifying motional electromagnetic fields, and these are difficult to detect using passive geophysical techniques like magnetotellurics. A very limited amount of controlled source information indicates the presence of a highly resistive region near the surface of the oceanic lithosphere [Cox et al.,
1986], minimizing the effect of the Earth on motional induction. However, it should be noted that the lithosphere in the offshore EMSLAB area is extremely young ( $\leq 10 \mathrm{Ma})$, correspondingly hot, and probably more conductive than that studied by Cox et al., and extrapolation of a conductivity profile from an older region is risky. No independent water velocity measurements were collected during EMSLAB, so a direct calibration is not available. All velocities from the horizontal electric field in this paper will be quoted ignoring current leakage effects. However, the interaction correction is unlikely to exceed $10 \%$ based on geophysically reasonable models for the lithospheric conductivity.

The raw east electric field time series in the EMSLAB data were processed with a low-pass prolate filter having a 3-dB point at a period of 4 days and $60 \mathrm{~dB}$ of stopband attenuation. Figure 7 compares hourly mean values of these filtered data sets for six of the sites. It should be remembered that the motional east electric field is proportional to the north or meridional water velocity; since the North American coast strikes nearly north-south, this is the longshore component. The top pair of series are spaced about $40 \mathrm{~km}$ apart east-west at the northwest comer of the array and straddling the Juan de Fuca Ridge. A quasi-sinusoidal component with a period near 4 days is quite apparent and correlates well between sites, as does the sharp increase at about 6300 hours. The middle pair of series are from two sites along the central line of Figure 1, close to the coast, and separated by about $25 \mathrm{~km}$. While some visual coherence is seen, it is not as high as for the last pair, and the periodic term is not present. The bottom pair of series are from the southern line and are separated by about $220 \mathrm{~km}$ eastwest. There is little visual correlation, and site SG4 near the Juan de Fuca Ridge shows a periodic term similar to that in the top pair of time series. The large electric field change seen at SG2 with a characteristic time scale of about 1000 hours or 40 days may be due to a barotropic mesoscale eddy. Note that the north-south 

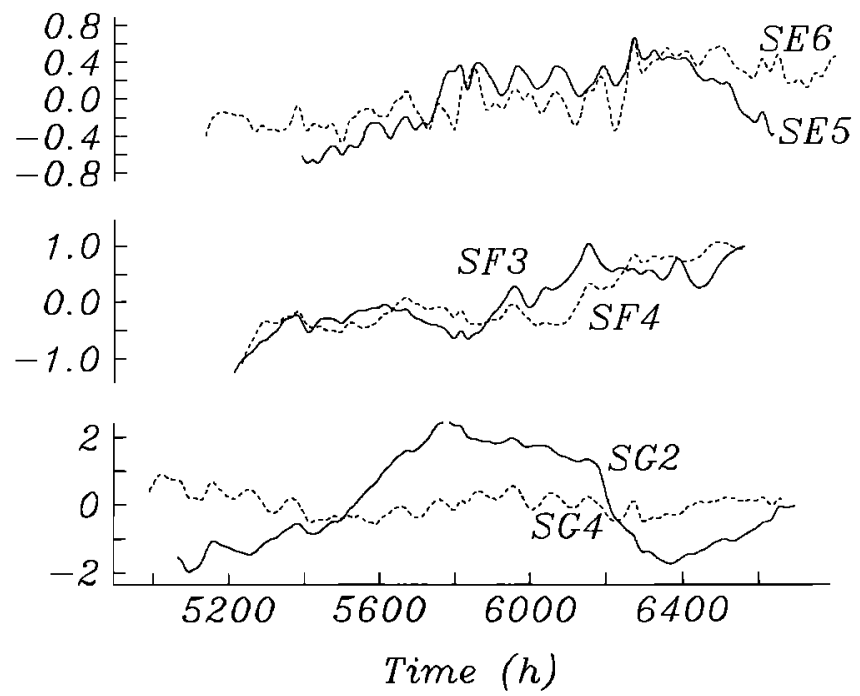

Fig. 7. The geographic east electric field time series for six stations after low passing with a prolate filter having a 3-dB point at 4-days period. The abscissa is the time in hours from 0000 UT on January 1, 1985, while the ordinate is the electric field in $\mu \mathrm{V} \mathrm{m}^{-1}$ and has a different scale for each of the three pairs of stations. In the absence of electromagnetic interactions with the conducting earth, the equivalent north depth-averaged velocities in $\mathrm{cm} \mathrm{s}^{-1}$ are obtained by scaling the electric field with a factor of 2 .

correlations of these data are not high, as seen by comparing the different pairs of time series.

Figure 8 contrasts the low-pass filtered vertical electric field time series from site SF7 with the similarly processed geomagnetic north electric field component at the same location. Both of these quantities give the horizontal velocity positive to the west. The visual correlation of these two data series is not high, reflecting the substantial difference between a depth-averaged and a point measurement of the water velocity. The vertical electric field is dominated by the baroclinic velocity at the seafloor, while the horizontal electric field accentuates the barotropic one. A quasi-sinusoidal signal like that of Figure 7 is weakly apparent in the vertical electric field but not in its horizontal counterpart, suggesting that the process producing it is more energetic near the seafloor. A coherence spectrum between these two series (not shown) is not significantly different from zero except in narrow bands centered at $0.02 \mathrm{cph}$ and $0.01 \mathrm{cph}$.

\section{SOME DETAILS OF THE LONG-PERIOD ELECTRIC FIELD}

The long-period electric field displays more complex behavior than the magnetic field, some of which is apparent in Figures 7 and 8. For this reason, a few intersite electric field coherences analogous to Figure 5 fail to illustrate the diversity of phenomena contained in the EMSLAB data. Instead, a more detailed look at a few specific characteristics of the electric field will be made.

The most prominent signal in Figure 7 is the nearly periodic 4day component in the horizontal electric field at sites SE5, SE6, and SG4. This component is also seen at site SF8 and very weakly at site SF7; these measurements are the closest ones to the topographic high associated with the Juan de Fuca spreading center. Figure 9 shows power spectra of the horizontal electric field at sites SE5 and SG4 using the rotary representation that was outlined earlier. Since the long-period electric field is a proxy for the depth-integrated water velocity, the complex time series that were analyzed had minus the north component in the real part and the east component in the imaginary part; these are appropriate for the east and north velocity components, respectively, the usual $\hat{x}$ and $\hat{y}$ coordinates used in oceanography. This means that the sense of rotation in the figures will be correct for the water velocity and reversed for the actual electric field vector; this convention will be used for the remainder of this paper. At high frequencies $(>0.02 \mathrm{cph})$, the clockwise and counterclockwise rotating parts have similar amplitudes, reflecting a roughly linearly polarized external source field. There is a slight tendency to an excess of clockwise-polarized (in the sense of the electric vector) energy near $0.4 \mathrm{cph}$. At lower frequencies, both sites show an unpolarized increase in power toward low frequency. Superimposed on this is a predominantly clockwise-polarized (in a velocity sense) enhancement of variance centered near four days period. It is important to note that the spread of this feature reflects the resolution bandwidth of the multiple window spectrum rather than the fundamental bandwidth of the process producing it. An $F$ test using the method of Thomson [1982] fails; the peak in the spectrum represents a narrowband process rather than a quasideterministic one with a constant phase relationship. At site SE5 the motion is essentially circular, as reflected in the total absence of a variance increase in the counterclockwise polarization, while at SG4 the motion is more elliptical. The remaining ridge sites show nearly linear motion at SE6 and nearly circular motion at SF7 and SF8, although the signal level is reduced at the latter two points. Cannon and Pashinski [1987] report on a 3.8-day peak in current meter data from the southern end of the Juan de Fuca Ridge that may be related. No evidence for this 4-day signal could be detected in rotary spectra at the remaining electric field sites.

Figure 10 shows the variance or kinetic energy in the frequency band 0.008-0.014 cph, encompassing the spectral peaks in Figure 9 , obtained by direct integration of a complete set of rotary spectra as a function of distance from the nominal axis of the Juan de Fuca Ridge as delineated by the topographic high. After a crude correction for the dependence of adjacent frequencies in the multiple window spectra, these are $\chi^{2}$ distributed with about 42 degrees-of-freedom per value, yielding double-sided $95 \%$ confidence limits of $(0.7,1.6)$ times the estimate. There is a general tendency for increased energy near the coast and near the spreading ridge with a minimum near the center of the Cascadia Basin; there is no preferential polarization associated with this. However, there is a statistically significant enhancement in the clockwise energy near the ridge axis by a factor of 3-6 over midbasin values, suggesting a causative relationship with the topographic high. The rms water velocity is up to $0.20-0.30 \mathrm{~cm} \mathrm{~s}^{-1}$, about 5 times smaller than a typical open ocean barotropic tidal

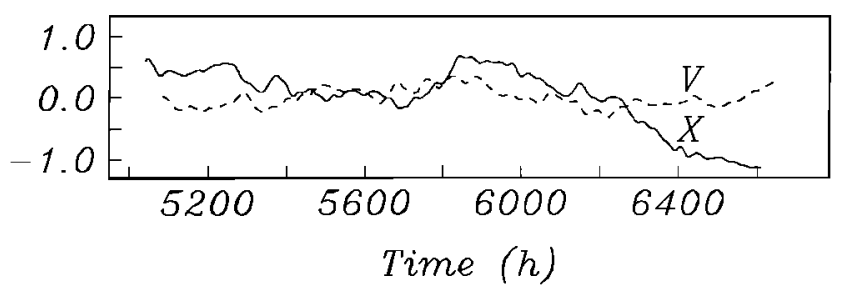

Fig. 8. The geomagnetic north (X) and vertical (V) electric components at site SF7 (solid triangle in Figure 1). The abscissa is the time in hours from 0000 UT on January 1, 1985, while the ordinate is the electric field in $\mu \mathrm{V}$ $\mathbf{m}^{-1}$. 

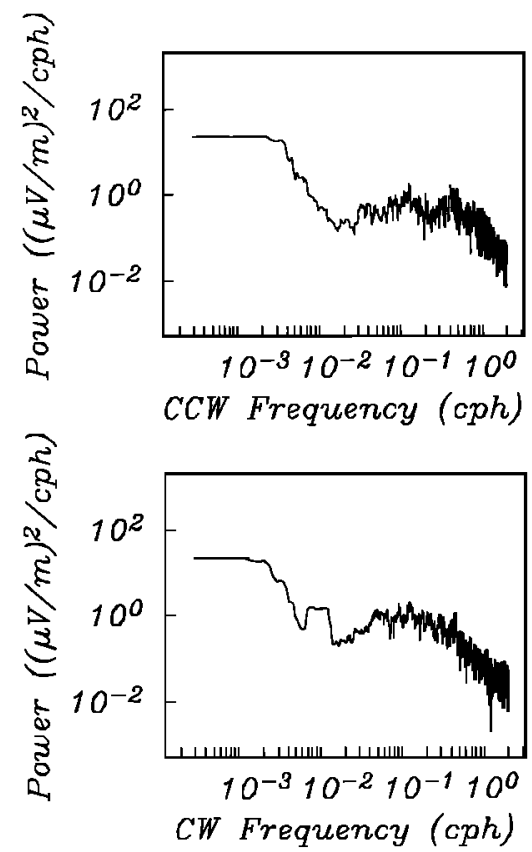
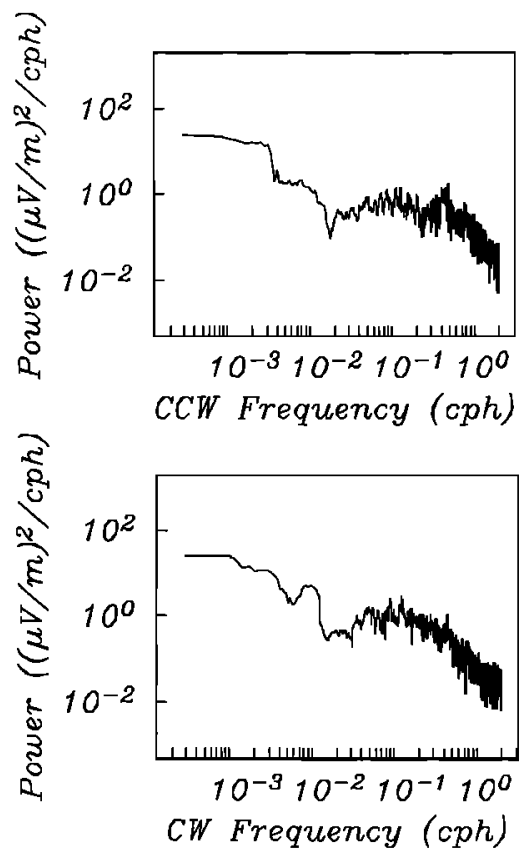

Fig. 9. Rotary multiple window power spectra at sites (left) SE5 and (right) SG4 with a time-bandwidth product of 4 and eight windows each, yielding about 16 degrees-of-freedom for $95 \%$ confidence limits of $(0.6,2.3)$ times the estimate. The spectral bandwidth is $\approx 0.0028 \mathrm{cph}$. The bottom panels show the clockwise-polarized part of the spectrum, while the top ones show the counterclockwise ones.

value. This figure is also smaller than the peak-to-peak value in Figure 7 would suggest due to the non-steady state nature of the 4-day wave.

Figure 11 shows two-point rotary coherence and phase plots for
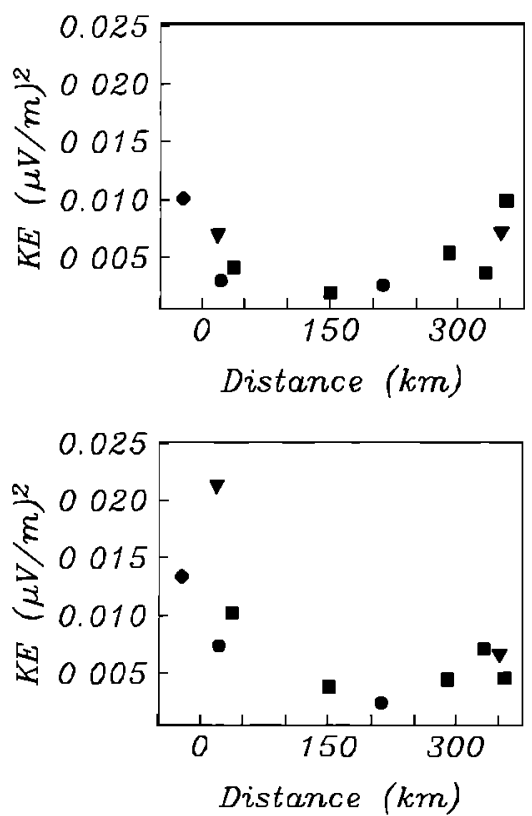

Fig. 10. Kinetic energy obtained by integrating rotary multiple window spectra over the frequency interval $0.008-0.014 \mathrm{cph}$, corresponding to the low-frequency peaks in Figure 9, as a function of distance from the topographic high associated with the Juan de Fuca Ridge. The clockwise polarization is shown in the bottom panel, while the counterclockwise one is at the top. The symbols correspond to the three east-west lines of Figure 1 with curcles for the $\mathrm{E}$ line, squares for the $\mathrm{F}$ line, and triangles for the $\mathrm{G}$ line. Each value is approximately $\chi^{2}$ distributed with 42 degrees-offreedom, yielding double-sided $95 \%$ confidence limits of $(0.7,1.6)$ times the estimate. the horizontal electric fields at site SE5 with respect to those at SF8. The coherence is high and the signals are linearly polarized at frequencies above $0.02 \mathrm{cph}$, with the usual holes seen in the coherence at the frequencies of $S_{q}$ and its first two harmonics. The coherence drops sharply at $0.02 \mathrm{cph}$ (2-day period) and rises again at the lowest resolvable frequencies $(<0.005 \mathrm{cph})$. The coherence is not different from zero between 0.005 and $0.02 \mathrm{cph}$ except for a narrow peak in the clockwise polarization at 0.01 $0.014 \mathrm{cph}$. The phase is about $20^{\circ}$ over this interval, and its sense suggests wave propagation from north-to-south. Similar analyses have been performed for the remaining pairs of sites on the east flank of the ridge. The phase consistently indicates north-to-south propagation along the east flank of the Juan de Fuca Ridge, although its size is not consistent with the propagation of a single plane wave. However, the rotary phase angle reflects both a temporal difference for a propagating component and a relative difference in the orientation of the polarization ellipses at the two sites. These can be distinguished in principle following Mooers [1973], but attempts to do so were not successful. This may indicate the presence of more than one component to the signal, as is also suggested by the persistent occurrence of a second peak with a larger phase at lower frequencies $(0.008-0.01 \mathrm{cph})$ for the three east flank sites (SE5, SF8, and SG4); see the top left panel of Figure 11 for an example. While the coherence is not significant over this interval, it should be remembered that a small amount of destructive interference associated with a mixture of wavenumbers will drive the coherence magnitude down very quickly without strongly influencing its phase. A best estimate for the dominant wavelength of the propagating component is $1000 \mathrm{~km}$, although the uncertainty is up to a factor of 2 . This gives a phase velocity along the ridge of about $3 \mathrm{~m} \mathrm{~s}^{-1}$. If there is also a shorterwavelength component at longer periods, then its phase velocity is much smaller.

This relatively simple picture for the ridge's east flank is complicated somewhat by including site SE6 on the west side. It was 

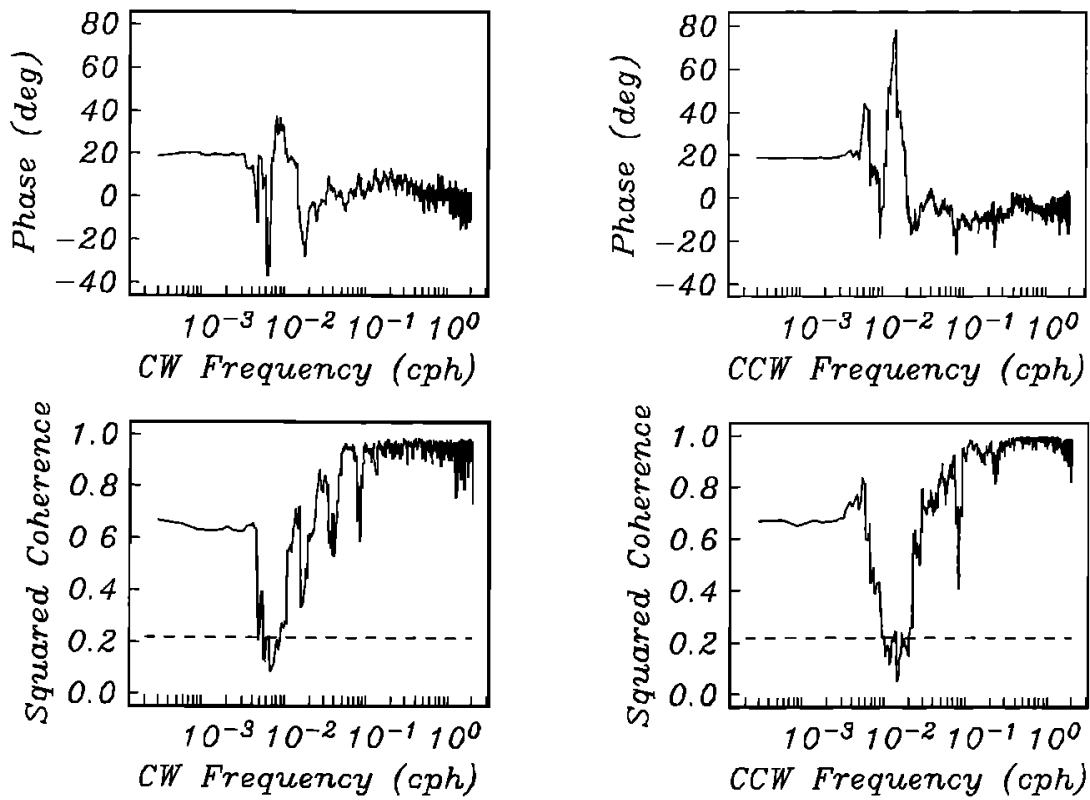

F1g. 11. Rotary multiple window two-point coherences for the horizontal electric field at sites SE5 and SF8, located on the east flank of the Juan de Fuca spreading center. The left panels show the clockwise-polarized component, while the counterclockwise one is at the right. The spectra have a time-bandwidth of 6.5 and 13 prolate windows were used, so there are about 26 degrees-offreedom per frequency. The zero coherence level is shown by the horizontal dashed line, while the spectral bandwidth is $\approx 0.0045$ cph. A positive phase angle means that site SF8 is leading site SE5.

noted earlier that spectra indicate that the 4-day peak is nearly linearly polarized at that site. Cartesian coherences in a geomagnetic coordinate system, which is nearly aligned with the strike of the ridge axis, indicate a high $\left(\gamma^{2} \approx 0.7\right)$ value for the north electric component with no phase lag and a somewhat smaller one $\left(\gamma^{2} \approx 0.45\right)$ for the east electric field with SE5 lagging SE6 by about $40^{\circ}$. This suggests a cross-ridge current that is in phase and phase propagation from west-to-east for the along-ridge component. A similar picture can be obtained by comparing SE6 and SF8.

The properties of the 4-day wave are suggestive of a topographically trapped Rossby wave. Rossby or planetary waves are a fundamental means by which energy is transported in the ocean and atmosphere at subinertial frequencies. Only some key properties of Rossby waves will be summarized here; see Gill [1982, chapter 12] for a detailed discussion of the theory underlying them. Rossby waves exist as a consequence of the conservation of angular momentum on a rotating earth and reduce to steady currents in the absence of rotation. A fundamental property of a rotating fluid in the absence of friction is the conservation of its potential vorticity, which can be expressed in the form

$$
\partial_{i}\left[\frac{\zeta+f}{H}\right]=0
$$

where $\zeta=(\nabla \times \vec{v}) \cdot \hat{z}$ is the vertical component of the fluid relative vorticity and $f=f_{o}+\beta y$ is the vertical component of the planetary vorticity caused by the rotation of the Earth. The latter is broken down into a value at a central latitude $\lambda$ given by $f_{o}=2 \Omega \sin \lambda$ and a linear variation in the north-south or $\hat{y}$ direction with $\beta=2 \Omega a^{-1} \cos \lambda$, where $\Omega$ is the angular rotation frequency of the Earth and $a$ is its radius. The term in brackets is the potential vorticity $Q$, and (4) simply states that it is a constant in time. In a flat-bottomed ocean where $H$ does not change, any north-south perturbation of the fluid will change $f$, and hence the relative vorticity by virtue of (4). The fluid will rotate in an anticlockwise direction if displaced toward the equator and in a clockwise direction if displaced poleward, setting up a wavelike motion called a Rossby wave. Figures $12 a$ and $12 b$ illustrate this. This type of Rossby wave always has a component of its phase velocity to the west and is dispersive, having a group velocity that is neither parallel nor perpendicular to the phase velocity. The shortestperiod mode is the barotropic (depth-independent) one with a cutoff at about 5 days period and a wavelength of about $1000 \mathrm{~km}$ at mid-latitudes. Baroclinic (depth-dependent) Rossby waves have both longer periods and shorter wavelengths.

In the presence of topography, this simple picture must be altered. Instead of a latitude deflection of a fluid parcel, consider a perturbation which moves it up or down a topographic slope as in Figures $12 c$ and $12 d$. From (4), relative vorticity will be imparted to the fluid such that displacement into deep water gives anticlockwise motion and into shallow water yields clockwise rotation. This results in wave propagation along a topographic contour with shallow water to the right.

It can be shown that the topographic effect is much larger than the planetary effect if the bottom slope exceeds 1 part in $10^{3}$, and only the former need be considered here. The approximate dispersion relation for a topographic Rossby wave on a uniformly sloping bottom is

$$
\omega=-\frac{\alpha f_{o} \eta}{H_{o}\left(\eta^{2}+\xi^{2}\right)}
$$

where $\alpha$ is the topographic slope, $H_{o}$ is a reference depth, and $\eta$ and $\xi$ are the two wavenumber components. For the large-scale topography of the Juan de Fuca Ridge, $H_{o} \approx 2800 \mathrm{~m}$ and $\alpha=600$ $\mathrm{m} / 100 \mathrm{~km} \approx 6 \times 10^{-3}$. To demonstrate order of magnitude consistency with the observations, take $f_{o} \approx 10^{-4}$ at $45^{\circ} \mathrm{N}$ and consider $\eta \approx \xi \approx 2 \pi / 1000 \mathrm{~km}^{-1}$, yielding $\omega \approx 2 \times 10^{-5}$ or a period of 87 hours. The observed phase propagation direction on the east flank of the ridge with shallow water on the right is consistent with 


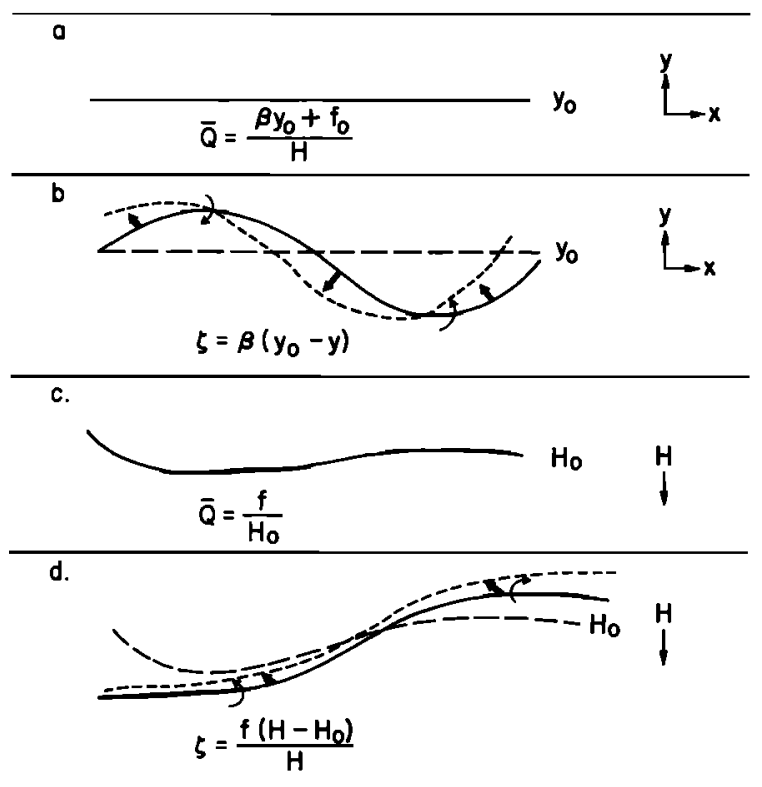

Fig. 12. Cartoon illustrating Rossby wave behavior given the conservation of potential vorticity in equation (4). Figure $12 a$ shows a parcel of fluid at equilibrium at latitude $y_{o}$ in an ocean of constant depth $H$, so the initial potential vorticity is just the planetary value $\bar{Q}=\left(\beta y_{o}+f_{o}\right) / H$. If the fluid is perturbed such that its distribution with latitude is altered, then it will acquire relative vorticity $\zeta$ such that the potential vorticity (4) is conserved. Equatorward deflection will result in anticlockwise rotation of the fluid, while polar motion gives a clockwise sense. This results in a Rossby wave motion propagating to the west as show by the large arrows. The analogous situation in the presence of topography is shown in Figure 12d. A parcel of fluid at equilibrium and distributed along a line of constant water depth $H_{o}$ has only a planetary part to the potential vorticity. If a perturbation from this situation is hypothesized, then relative vorticity is acquired by the changing water depth. If the water gets deeper so that the fluid column stretches, then anticlockwise motion results. The result is a topographic Rossby wave moving along the bottom contour such that shallow water lies to the right of the direction of phase propagation. topographic Rossby wave behavior. It can also be shown that for topography such that $\alpha / H_{o}$ is bounded, the group velocity will change sign at some location in frequency-wavenumber space [Huthnance, 1975]. This means that a group velocity zero must exist at which no energy propagates but where phase propagation is still possible. This suggests a working hypothesis to explain the observations: some external source, possibly atmospheric forcing, is pumping energy into the ocean at the appropriate spatial scale to excite depth-independent or weakly depth-dependent currents. This energy is quickly transported away from the forcing region except at the resonancelike group velocity minimum, where the very long-lived phase propagation necessary to explain the observations could occur.

As a second illustration of the diverse phenomena present in the electric field data, Figure 13 shows the Cartesian coherences and phases between sites SE3 and SG2, spanning a north-south distance of $180 \mathrm{~km}$ in the center of the Cascadia Basin. The coherence is high at frequencies above $0.03 \mathrm{cph}$, especially for the north component, but there is a fairly complex phase structure due to source field effects. The sense of the phase is such that the south site (SG2) leads the north one (SE3), and its size increases with period. The coherence falls rapidly at frequencies below $0.02 \mathrm{cph}$, but a very sharp peak occurs over $0.0035-0.005 \mathrm{cph}(8$ to 12 day period) in both electric field components. There is a distinct phase associated with both of these peaks, suggesting a north-to-south propagating component with a wavelength of about $1000 \mathrm{~km}$. This peak is seen for several pairs of stations in the middle of the basin, but is not clear when either near-ridge or near-coast ones are included, although this may indicate interference from unresolved low-frequency components within a spectral bandwidth of zero frequency. The origin of this propagating wave is not known, but it is interesting to speculate about open ocean barotropic wave phenomena. Such waves could be observed in the absence of topography but would be obscured by others having a multiplicity of wavenumbers near the coast or the ridge.
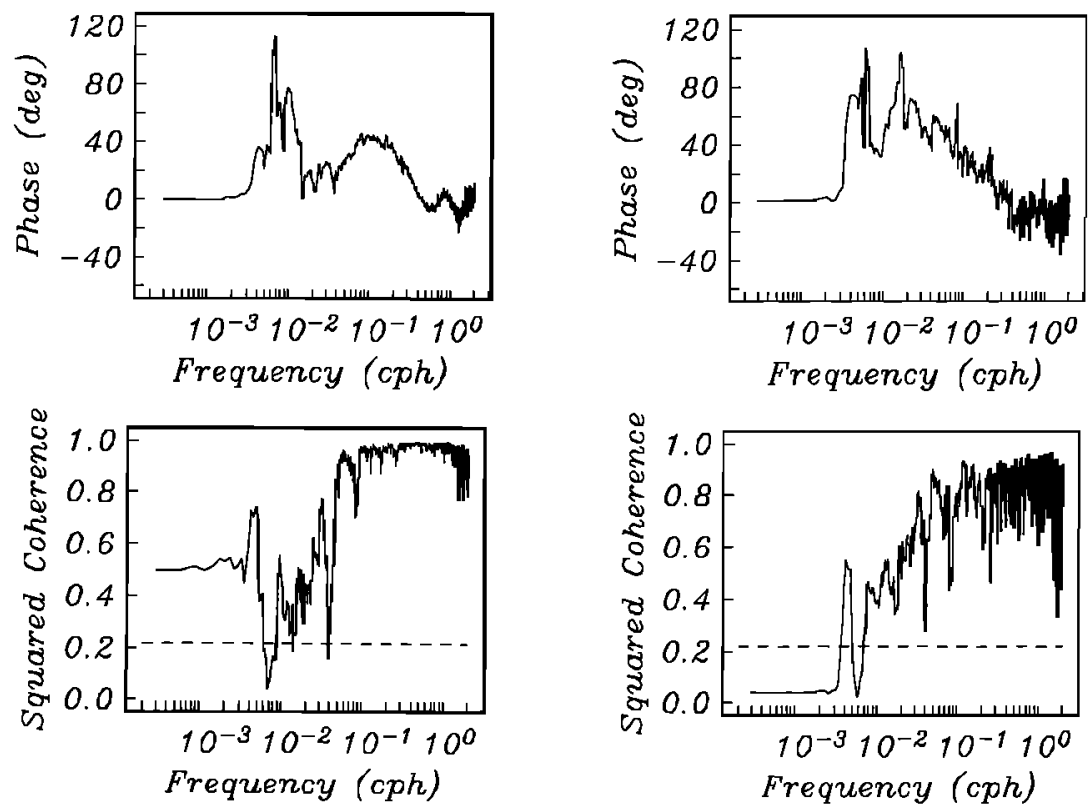

Fig. 13. Cartesian multiple window coherences and phases for the horizontal electric field at sites SE3 and SG2, spanning the middle of the Cascadia Basin from north-to-south. The north electric components are shown in the left panels, while the east ones are at the right. The spectra have a time-bandwidth product of 6.5 and 13 prolate data windows were used, yielding about 26 degreesof-freedom per frequency. The zero coherence level is shown by the horizontal dashed line, while the spectral bandwidth is $\approx$ $0.0045 \mathrm{cph}$. A positive phase corresponds to site SG2 leading site SE3. 

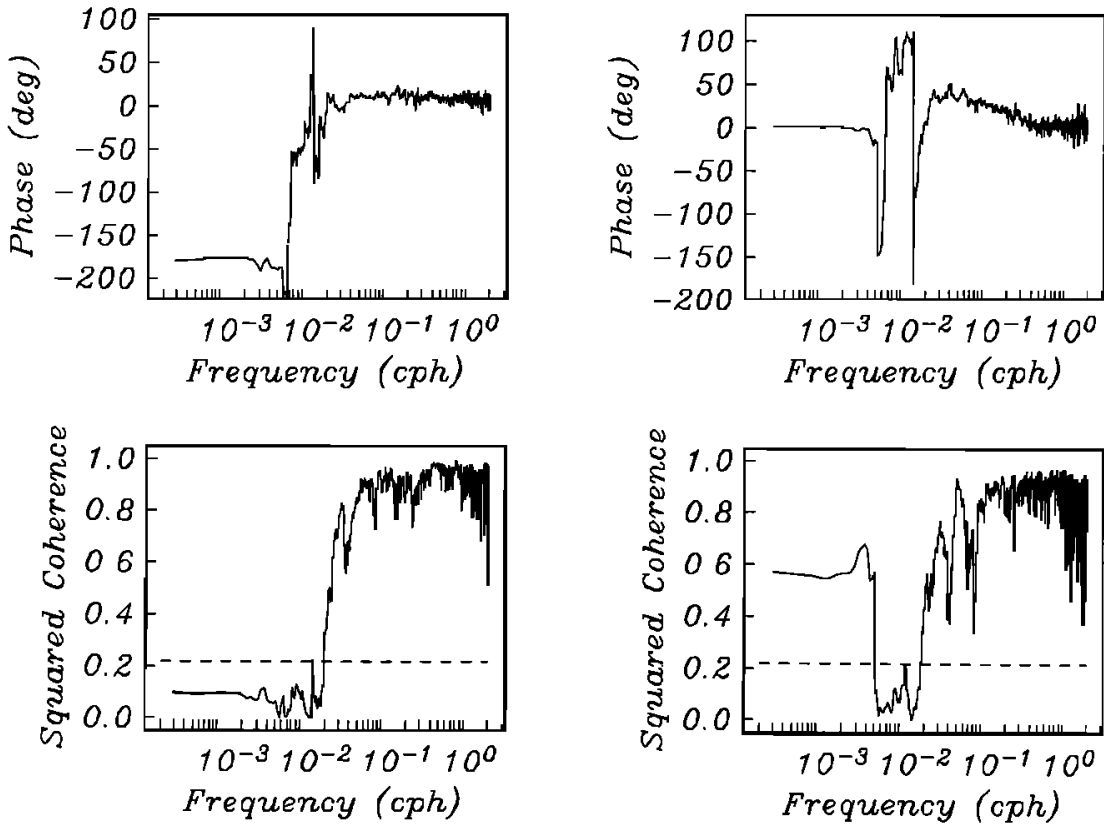

Fig. 14. Cartesian multiple window coherences and phases for the horizontal electric field at sites SF8 and SF3, spanning the middle of the Cascadia Basin from east-to-west. The north electric components are shown in the left panels, while the east ones are at the right. The spectra have a time-bandwidth product of 6.5 and 13 prolate data windows were used, yielding about 26 degreesof-freedom per frequency. The zero coherence level is shown by the horizontal dashed line, while the spectral bandwidth is $\approx$ $0.0045 \mathrm{cph}$. A positive phase corresponds to site SF3 leading site SF8.

Figure 14 shows a Cartesian two-point coherence for the eastwest separated measurements at SF8 and SF3 along the middle line of Figure 1. The intersite distance is about $320 \mathrm{~km}$. The coherence is high for frequencies above $0.02 \mathrm{cph}$, falling rapidly below this, and never rising above zero for the north electric field. The east electric field coherence rises quickly again at low frequencies. Similar behavior is seen for the north electric field component in Figure 13. This unresolved low-frequency component appears at many locations in the EMSLAB data, but it is difficult to establish a clear pattern, probably reflecting the presence of several independent processes at different frequencies that are mixed together during analysis because of resolution problems. A longer time series would allow these to be studied.

Further study of the offshore EMSLAB data for oceanographic purposes is certainly warranted. For example, surface wind data computed from ship observations are available throughout the area and can be used to establish causative relationships, if any, between atmospheric forcing and the depth-averaged currents measured by the horizontal electric field. There are also five seafloor pressure records at sites SE3, SE5, SG2, SG3, and SG4 that may provide important clues about some of the phenomena described here. This report should be regarded only as a preliminary description of the oceanic effects on the seafloor electromagnetic fields during EMSLAB and further, more quantitative treatments will be forthcoming.

Acknowledgments. EMSLAB data collection and analysis at SIO was supported by the National Science Foundation under EAR85-12895. The authors are grateful to Greg Neumann of Brown University for providing the Victoria Observatory data. This is Geological Survey of Canada contribution 52188 .

\section{REFERENCES}

Bindoff, N. L., J. H. Filloux, P. J. Mulhearn, F. E. M. Lilley, and I. J. Ferguson, Vertical electric field fluctuations at the floor of the Tasman abyssal plain, Deep Sea Res., 33, 587-600, 1986.
Cannon, G. A., and D. J. Pashinski, Mid-depth flow near a venting site on the Juan de Fuca Ridge (abstract), Eos Trans. AGU, 68, 1306, 1987.

Chave, A. D., On the electromagnetic fields induced by oceanic internal waves, J. Geophys. Res , 89, 10519-10528, 1984.

Chave, A. D., and J.H. Filloux, Electromagnetic induction fields in the deep ocean off California: Oceanic and ionospheric sources, Geophys. $J$. R. astron. Soc., 77, 143-171, 1984.

Chave, A. D., and J.H. Filloux, Observation and interpretation of the seafloor vertical electric field in the eastern North Pacific, Geophys. Res. Lett., 12, 793-796, 1985.

Chave, A. D., D. J. Thomson, and M.E. Ander, On the robust estimation of power spectra, coherences, and transfer functions, J. Geophys. Res, 92, 633-648, 1987.

Cox, C. S., S. C. Constable, A. D. Chave, and S. C. Webb, Controlledsource electromagnetic sounding of the oceanic lithosphere, Nature. $320,52-54,1986$.

EMSLAB Group, The EMSLAB electromagnetic sounding experiment, Eos Trans AGU, 69, 89, 98-99, 1988.

Filloux, J. H., Instrumentation and experimental methods for oceanic studies, in Geomagnetism, vol. 1, edited by J. A. Jacobs, pp. 143-248, Academıc, San Diego, 1987.

Fu, L.-L., Observations and models of inertial waves in the deep ocean Rev. Geophys., 19, 141-170, 1981.

Gill, A. E., Atmosphere-Ocean Dynamics, Academic, San Diego, 1982.

Huthnance, J. M., On trapped waves over a continental shelf, J. Fluid Mech., 69, 689-704, 1975.

Larsen, J. C., Electric and magnetic fields induced by deep sea tides, Geophys. J R. astron. Soc., 16, 47-70, 1968

Larsen, J. C., and T. B. Sanford, Florida Current volume transports from voltage measurements, Science, 227, 302-304, 1985.

Lilley, F. E. M., J. H. Filloux, N. L. Bindoff, I. J. Ferguson, and P. J. Mulheam, Barotropic flow of a warm-core ring from seafloor electric measurements, J. Geophys. Res., 91, 12,979-12,984, 1986.

Luther, D. S., A. D. Chave, and J. H. Filloux, BEMPEX: A study of barotropic ocean currents and lithospheric electrical conductivity, Eos Trans. $A G U, 68,618-619,628-629,1987$.

Mooers, C. N. K., A technique for the cross spectrum analysis of pairs of complex-valued time series, with emphasis on properties of polarized components and rotational invariants, Deep Sea Res., 20, 1129-1141, 1973.

Sanford, T. B., Motionally induced electric and magnetic fields in the sea, J. Geophys. Res , 76, 3476-3492, 1971. 
Sanford, T. B., Temperature transport and motional induction in the Florida Current, J. Mar. Res, 40 (suppl.), 621-639, 1982.

Schultz, A., and J. C. Larsen, On the electrical conductivity of the midmantle, I, Calculation of equivalent scalar magnetotelluric response functions, Geophys J.R. astron. Soc., 88, 733-761, 1987.

Thomson, D. J., Spectrum estimation and harmonic analysis, Proc. IEEE, 70, 1055-1096, 1982.

White, A., A sea floor magnetometer for the continental shelf, Mar. Geophys. Res., 4, 105-114, 1979.

Wunsch, C., Low-frequency variability of the sea, in Evolution of Physical Oceanography, edited by B. A. Warren and C. Wunsch, pp. 342-375, MIT Press, Cambridge, Mass., 1981.

A. D. Chave, AT\&T Bell Laboratories, 600 Mountain Ave., Murray Hill, NJ 07974
J. H. Filloux and D. S. Luther, Scripps Institution of Oceanography, La Jolla, CA 92093

L. K. Law, Geological Survey of Canada, Pacific Geoscience Center, Sidney, B.C. V8L 4B2, Canada

A. White, School of Earth Sciences, The Flinders University of South Australia, Bedford Park, South Australia 5042, Australia
(Received July 18, 1988; revised March 15, 1989; accepted March 21, 1989.) 